\title{
Mate Analysis of Comprehensive Treatmentstrategy for Advanced Rectal Cancer
}

\author{
Huaikun $\mathrm{Ni}^{1}$, Ruolei Huang ${ }^{1}$, Lianming Liao ${ }^{2}$, Wenjuan $\mathrm{Zhou}^{2, * *}$ \\ ${ }^{1}$ General Surgery Department, Fujian Provincial Hospital South Branch, Fuzhou, China \\ ${ }^{2}$ Central Laboratory, Union Hospital Affiliated to Fujian Medical University, Fuzhou, China
}

Email address:

821554100@qq.com (Wenjuan Zhou)

${ }^{*}$ Corresponding author

\section{To cite this article:}

Huaikun Ni, Ruolei Huang, Lianming Liao, Wenjuan Zhou. Mate Analysis of Comprehensive Treatmentstrategy for Advanced Rectal Cancer. International Journal of Clinical Oncology and Cancer Research. Vol. 5, No. 4, 2020, pp. 93-104. doi: 10.11648/j.ijcocr.20200504.13

Received: November 3, 2020; Accepted: November 26, 2020; Published: December 4, 2020

\begin{abstract}
AIM: Objective to evaluate the clinical value of therapeutic lateral lymph node dissection in advanced rectal cancer after neoadjuvant radio-chemotherapy in patients with peritoneal retroperitoneum under MR suggesting perivascular iliac lymph node enlargement (lymph node diameter $>8 \mathrm{~mm}$ ). Methods: Because of this study is a retrospective study, the propensity to evaluate matching group is adopted to one group to Fujian Medical University Union Hospital, Fujian province hospital from January 2009 to June 2014 treated 103 cases of rectum MR images after neoadjuvant chemoradiation weeks iliac blood vessels have the progress of the lymph node enlargement period under peritoneal fold return rectal cancer line TME + therapeutic cases of lateral lymph node cleaning; In the group 2, 192 cases with the same tumor site, stage, type, gender and age as group 1 or similar were matched for the same period by propensity assessment, while the group 2 received TME only. The incidence of pelvic autonomic nerve injury after surgery, the 3-year local recurrence rate of pelvic cavity, and the 5-year tumor-free survival rate were compared between the two groups, so as to explore the clinical value of therapeutic periiliac dissection for advanced rectal cancer under peritoneal reflux. Results: The local recurrence rate of pelvic cavity in group 1 and group 2 was $2.91 \%$ $(3 / 103)$ and $9.90 \%(19 / 192)$, respectively, 3 years after operation $(\mathrm{P}<0.05)$. Postoperative dysuria in group 1 and group 2 was $14.56 \%(15 / 103)$ and $5.21 \%(10 / 192)$, respectively, with significant difference $(\mathrm{P}<0.05)$. Postoperative sexual dysfunction in group 1 and group 2 was $12.50 \%(7 / 56)$ and $2.15 \%(2 / 93)$, respectively, with significant difference $(\mathrm{P}<0.05)$. The 5 -year survival rates of group 1 and group 2 were $54.37 \%$ (56/103) and 51.56\% (99/192), respectively, with no significant difference $(\mathrm{P}>0.05)$. The liver metastasis rates of group 1 and group 2 were $26.21 \%(27 / 103)$ and $29.17 \%(56 / 192)$, respectively. Lung metastasis rates in group 1 and group 2 were 19.41\% (20/103) and 19.27\% (37/192), respectively. Conclusion: After neoadjuvant chemoradiotherapy for advanced rectal cancer, selective lymphatic dissection along the iliac vessels guided by rectal magnetic resonance can reduce the local pelvic recurrence of patients for 3 years, but increase the probability of pelvic autonomic nerve injury, and periiliac lymph node dissection will not increase the 5 -year survival rate of patients.
\end{abstract}

Keywords: Rectal Cancer, Progressive Stage, n-RCT, Lateral Lymph Node Dissection

\section{Background and Foreword}

Nowadays, for advanced rectal cancer under peritoneum reflux, the choice of comprehensive treatment and surgical method is still controversial. Thorough lymph dissection may reduce the local recurrence rate of tumor pelvic floor, and rectal cancer above peritoneum reflux is generally treated as colon cancer [1]. Peritoneal fold return for near the anus rectum, under its anatomical structure and lymph flow path and peritoneal fold back on rectal recently, peritoneal reflected on the recent similar to the colon and bowel loops are serous membrane surrounding the ventral bilateral, lymphatic flow in the inferior mesenteric vascular lymph node to the abdominal aorta weeks lymph node flow is given priority to. Under peritoneal reflux, only the ventral serous membrane of the intestine exists in the rectum, and the visceral layer and parietal layer of the pelvic cavity are surrounded by peritoneum on both sides and dorsal side, and the surrounding tissues are mesenteric, containing a large amount of lymphatic adipose tissue [2]. Pelvic 
lymph node dissection studies have shown that under the peritoneal fold return the rectum lymphatic flow in addition to the upward direction path, peritoneal fold return under the existence of bilateral rectum rectal cancer blood vessels, iliac blood vessels to the lymphatic flow path, rectum lateral lymph flow is divided into three directions: 1 with bladder reproductive organ lymph flow to the pelvic floor front wall lymphatic, again to the internal iliac vessels weeks lymph nodes; 2 . The lateral direction passes through the pelvic plexus nerve network and crosses the internal iliac blood vessel to the obturator pore peripheral lymph node; 3 . It flows posteriorly through external iliac vessels and anterior sacral vessels to lymphatic vessels in the posterior wall of the pelvic cavity. In addition, under peritoneal reflux, the rectum has a confluence of perineal lymphatics down to the superficial inguinal lymph nodes, and further back to the deep inguinal lymph nodes, the periiliac lymph nodes. Progress in [3] period under peritoneal fold return rectal tumor, iliac blood vessels in the recent weeks have Japanese scholars reported the probability of positive lymph nodes can reach $10 \%$, that pure line TME (Total Mesorectal Response) pelvic floor still exist the possibility of local recurrence.

Preoperative neoadjuvant chemoradiotherapy is routine for patients with advanced subperitoneal reentry rectal cancer. Long-term concurrent chemoradiotherapy was generally used, and radical surgery was performed 8 weeks later. For cases with positive lymph nodes around iliac vessels suggested by imaging, can neoadjuvant chemoradiotherapy replace iliac perivascular lymphatic dissection, the clinical value of iliac perivascular lymphatic dissection, and whether therapeutic lateral dissection can benefit disease-free survival of patients with advanced rectal cancer [3].

European and American scholars and Japanese and Korean scholars still have disputes about the lateral lymph node dissection. European and American scholars believe that neoadjuvant chemoradiotherapy can replace lateral lymph node dissection. After neoadjuvant chemoradiotherapy, the positive rate of lateral lymph node pathology in rectal cancer cases after surgery is very low, with only $1.9 \%$ reported in literatures [4-5]. Unless after neoadjuvant chemoradiation, rectal MRI prompted cases of iliac blood vessels weeks lymph node positive, then to iliac blood vessels of the limits of the lymph node weeks, European and American scholars advocate side cleaning and Japanese scholars point of view is different, the European and American scholars advocate clean to the internal iliac vessels weeks lymph nodes, they think that the internal iliac vessels distal segment spread to lymph nodes is far viscera, side cleaning does not solve the problem, have the literature major advanced colorectal cancer death for tumor metastasis of lung, liver and other organs apart, side cleaning does not improve the prognosis of patients. Moreover, lateral dissection significantly increased the risk of pelvic autonomic nerve injury in patients. The network structure of the pelvic autonomic nerve plexus was located on both sides of the rectum, and lymph node dissection along the obturator foramen inside and outside the iliac group greatly increased the probability of injury innervating the nerve branches of the bladder and the cavernous body of the penis. Iliac blood vessels, by contrast, a Japanese scholar think weeks lymph nodes are regional lymph nodes, a Japanese scholar reported iliac blood vessels weeks after surgery and pathology of lymph node positive rate can reach $20 \%$, JSCCR Colon Cancer association (Japan Japanese Society for Cancer of the Colon and Rectum) Suggestions for tumors located in the peritoneal fold back under rectal tumor, tumor invasion, leaching and serous T3-4 tumor, Rectum were recommended prophylactic lateral lymph node cleaning line, and at the same time cleaning the bilateral iliac blood vessels weeks lymphoid tissue [6]. A retrospective analysis of a large number of cases by Japanese scholars revealed that rectal tumor was located under peritoneum. After tumor involvement in rectal serous membrane, the rate of iliac perivascular lymph node metastasis reached $20.1 \%$, and lateral dissection reduced the 5 -year local recurrence rate of pelvic cavity by half, and achieved a 5-year survival rate of $8-9 \%$ [7]. However, the Japanese study did not include neoadjuvant chemoradiotherapy, which is the reason why European and American scholars did not approve the results of JSCCR.

Japanese scholar week of iliac blood vessels will be further divided into regional lymph node and remote lymph nodes, according to the latest version (version 8) colorectal treatment protocols, distal near side of the internal iliac vessels and lymph nodes, closed around for low rectal cancer in the lymph node of local regional lymph nodes, external iliac lymph nodes, common iliac lymph nodes as far, if appear external iliac, common iliac tumor metastasis is M1, therapy in locally advanced rectal cancer treatment [8]. National American cancer network NCCN and ESMO (European society of oncology) are only to the internal iliac vessels week of proximal lymph node is considered to be regional lymph nodes, believe that only after neoadjuvant chemoradiation, magnetic resonance imaging is still suspected proximal internal iliac vessels weeks will only be expand sweep of limitation and lymph node metastasis in order to avoid expand sweep bring urination and sexual function in disorder $[9,10]$. In pelvic cavity, the sympathetic and parasympathetic nerve fiber of pelvic autonomic nerve, 12 to thoracic lumbar 2 ganglia of sympathetic nerve fibers, the inferior mesenteric vessels weeks, before of sacral nerve in front of the abdominal aorta, and then to bilateral hypogastric nerves, on either side of the hypogastric nerves on either side of the line to join up the internal iliac vessels to the pelvic plexus, sympathetic nerve associated with male ejaculation. The parasympathetic nerve fibers of the sacral 2 to sacral 4 ganglion pass through the sacral foramen through the sacral anterior foramen and join the pelvic nerve plexus to form the pelvic visceral nerve, which is mainly responsible for bladder urination and male erectile function. Pelvic viscera nerve branches form a network of nerve fibers, which are distributed in an inverted triangle on both sides of the rectum [11]. According to local anatomy under peritoneal fold back on either side of the rectum, along the common iliac and iliac blood vessels within the bones of the lymph node cleaning is likely to bring the injury of pelvic autonomic nerve plexus, unilateral pelvic autonomic nerve plexus injury after a period of compensatory, micturition, male erectile, ejaculation function 
could recover, but if they are bilateral hypogastric nerve injury, may cause neurogenic bladder, need lifetime bladder fistula. How to avoid damaging the network - like structure of pelvic plexus is a difficult point when iliac perivascular lymph node dissection, which also tests the surgical level of colorectal surgeons.

At present, the lymph node cleaning of iliac blood vessels in the week is not perfect, advanced rectal cancer after neoadjuvant chemoradiation, based on the preoperative imaging accurate staging and positioning, iliac blood vessels such as found short weeks lymph node size more than $8 \mathrm{~mm}$, week of iliac blood vessels and lymph nodes with irregular shape, signal heterogeneity suspicious performance, such as expanding swept along the week of iliac blood vessels become advanced peritoneal lymph node reflected colorectal surgery under selection, accurate positioning the enlargement of lateral lymph node cleaning, on the one hand, can effectively reduce the pelvic tumor residual, on the other hand can avoid bilateral pelvic autonomic nerve damage, To avoid neurogenic bladder and erectile and ejaculation dysfunction [12]. However, the focus of this study is whether expanded dissection can bring about a change in 5-year survival rate of rectal cancer under progressive peritoneal reflux. Meanwhile, whether the therapeutic iliac vascular lymphatic dissection based on precise positioning according to preoperative imaging can effectively reduce the probability of pelvic autonomic nerve injury.

\section{Case Data and Analysis Methods}

\subsection{Inclusion Criteria for Group 1 and Group 2}

1. All cases were pathologically confirmed as rectal cancer, and no distant organ metastasis was found in the tumor.

2. Colonoscopy and surgery confirmed that the tumor was located under peritoneum reflux and above the dentate line.

3. All cases neoadjuvant chemotherapy, radiotherapy, or chemoradiation, tumor stage were yp III.

4. MR examination of the rectum was performed before surgery in all cases, and lymph nodes larger than $8 \mathrm{~mm}$ in diameter were found around the iliac vessels.

\subsection{The Exclusion Criteria of Group 1 and Group 2 Were the Same}

1. Early cases of rectal cancer.

2. Patients with original urinary system diseases accompanied by urination dysfunction.

3. Patients with severe cardiopulmonary dysfunction may affect the prognosis.

\subsection{Case Data of Group 1}

All cases 1 group were TME + lateral lymph nodes, TME for a dirty layer and the wall along the parcel rectal complete separation between the pelvic peritoneum, complete resection of tumor edge $5 \mathrm{~cm}$ above the mesorectum, incised side for more than $2 \mathrm{~cm}$ from the edge of the tumor, lateral lymph node cleaning for the order will be common iliac and iliac bone internal and external iliac blood vessels until the obturator foramen.

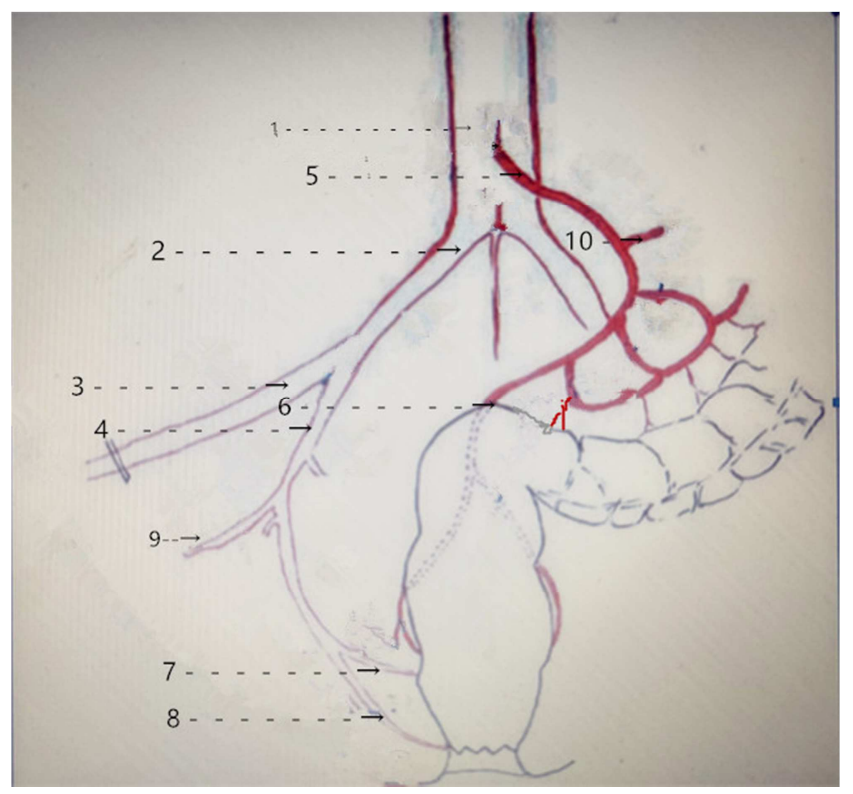

Figure 1. Schematic diagram of periiliac lymphatic dissection (the scope of the dissection includes sequential dissection of 2 total iliac vessels, 4 internal iliac vessels, 3 external iliac vessels, and 9 obturator perivascular lymphatic adipose tissue. 1. Aorta 2. Common iliac artery 3. External iliac artery 4. Internal iliac artery 5. Submesenteric artery 6. Superior rectal artery 7. Inferior rectal artery 8. Internal pudendal artery 9. Obturator artery 10. Left colonic artery) [13].

Cases: Group 1, from January 2009 to June 2014, 123 cases of advanced rectal cancer under therapeutic lateral lymphatic dissection were admitted and treated by Fujian Medical University Union Hospital and Fujian provincial hospital, among which 20 cases were excluded without neoadjuvant comprehensive treatment. A total of 103 patients underwent lateral lymph node dissection after neoadjuvant chemoradiotherapy. All patients underwent MR Imaging of the rectum (Magnetic Resonance Imaging), which indicated preoperative lymphadenopathy around the iliac vessels (lymph node short diameter $>8 \mathrm{~mm}$ ), tumor was located below peritoneal reflux and above the dentate line in the progressive stage, all patients received neoadjuvant chemotherapy or radiotherapy, FOLFOX or CAPOX chemotherapy regimen, and radiotherapy was 50-45Gy 25 times of irradiation. Among men and 56 cases of women and 47 cases, biggest 82 years old, smallest 32 , with an average age of $54+/-2.6$ years of age, preoperative imaging studies suggest tumor staging of T2-4 $\mathrm{n} 2 \mathrm{~m} 0$, based on preoperative imaging data, expand the scope of cleaning cleaning the corresponding lateral iliac blood vessels weeks of lymphoid tissue, assisted surgery procedures first improperness mirror colorectal cancer effect a radical cure (TME), then use ultrasonic knife order along the external iliac blood vessels inside the common iliac and iliac week to remove lymphoid tissue. Postoperative pathology revealed 31 cases of poorly differentiated adenocarcinoma, 16 cases of mucinous adenocarcinoma, 29 cases of moderately differentiated adenocarcinoma, and 27 cases of highly differentiated adenocarcinoma. The total number of pathological lymph nodes 
in all cases was more than 20, and 23 cases of iliac perivascular lymph node positive cases, including 3 cases of highly differentiated adenocarcinoma, 6 cases of moderately differentiated adenocarcinoma, 11 cases of poorly differentiated adenocarcinoma, and 3 cases of mucinous adenocarcinoma.

\subsection{Case Data of Group 2}

As this study is a retrospective study, the 2 groups of patients were matched with the 2 groups of patients in propensity assessment, and the tumor sites, stages, types, genders and ages in the same period were matched with those in groupl or similar. There were a total of 192 cases, including 93 males and 99 females, with the maximum age of 83 years and the minimum age of 31 years, and the average age was $54 \pm 2.3$ years.

The inclusion and exclusion criteria of the group 2 were the same as that of the group 1 . The special criteria of the 2 groups were TME surgery for all cases. TME was a complete separation between the visceral layer and the parietal pelvic peritoneum enclosing the rectum.

Cases: Group 2 for Fujian Medical University Union Hospital, Fujian province hospital between January 2009 and June 2014 have admitted progress period under the dentate line above, peritoneal fold return line rectal cancer after neoadjuvant chemoradiation tumor stage for yp III TME cases 1162 cases. To eliminate the relevant factors unrelated to side sweeping. Phase 2 set of selection bias matching progress under the dentate line above, peritoneal reflected rectal cancer cases, a total of 192 cases, two groups of 192 cases are all of two groups corresponding cases, gender, age and not more than 3 years of age, tumor were dentate line above, peritoneal fold back under, all cases of the corresponding tumor classification, all cases of rectum MR images hint weeks iliac blood vessels have swollen lymph nodes (lymph nodes short diameter holds $8 \mathrm{~mm}$ ), all cases were new adjuvant chemotherapy or radiation therapy, chemotherapy regimens for FOLFOX or CAPOX, radiotherapy for 50-45 Gy 25 points. Among them, 93 were male and 99 were female, the maximum was 83 years old and the minimum was 29 years old, with an average age of $54 \pm 2.3$ years old. Preoperative imaging suggested $\mathrm{T} 2-4 \mathrm{n} 2 \mathrm{~m} 0$, and the operation method was simple endoscopic assisted radical resection of rectal cancer (TME). Postoperative pathology showed 49 cases of low-differentiated adenocarcinoma, 23 cases of mucinous adenocarcinoma, 72 cases of moderately differentiated adenocarcinoma, and 48 cases of highly differentiated adenocarcinoma. All cases had more than 12 pathological lymph nodes. The matching conditions of the two groups and the two groups in propensity assessment are shown in table 1
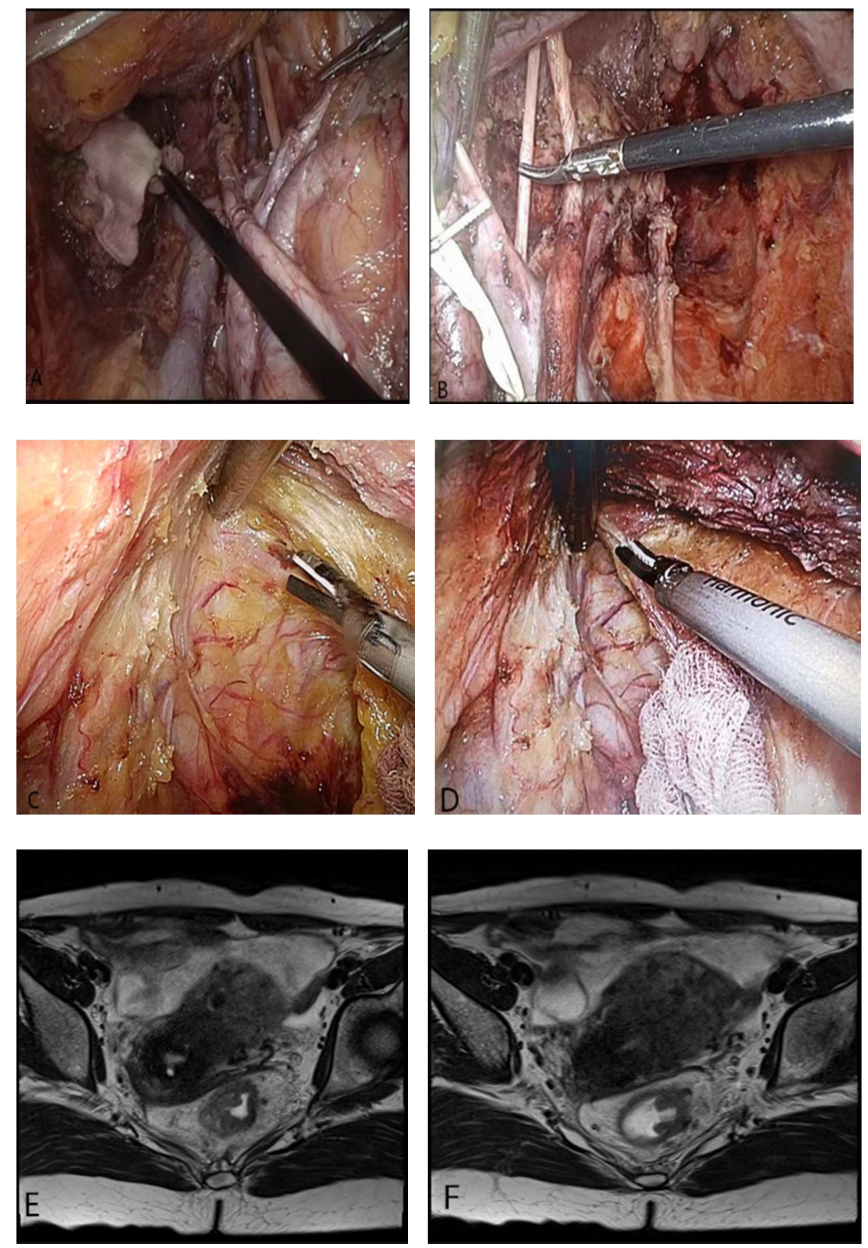

Figure 2. Case imsage data: figures $A$ and $B$ are lateral lymph node dissection images under endoscopy, which show complete dissection of lymphoadipose tissue to obturator foramen along the total iliac, internal iliac and external iliac vessels. Figures $C$ and $D$ are endoscope-assisted TME images, which show the complete free mesentery. Figure $E$ and figure $F$ are MR images of advanced rectal cancer with lateral lymph node enlargement.

Table 1. group 1 and group 2 were matched in propensity assessment

\begin{tabular}{|c|c|c|}
\hline Group & Group 1 (TME+ lateral group) & Group 2 (TME group) \\
\hline Tumor sites & $\begin{array}{l}\text { All below peritoneal reflux, and above the dentate line of } \\
\text { the anal canal }\end{array}$ & All below peritoneal reflux, and above the dentate line of the anal canal \\
\hline Tumor stage & ypIIIperiod & ypIIIperiod \\
\hline $\begin{array}{l}\text { Pathological } \\
\text { type of tumor }\end{array}$ & $\begin{array}{l}31 \text { cases of poorly differentiated adenocarcinoma, } 16 \\
\text { cases of mucinous adenocarcinoma, } 29 \text { cases of } \\
\text { moderately differentiated adenocarcinoma, and } 27 \text { cases } \\
\text { of highly differentiated adenocarcinoma. }\end{array}$ & $\begin{array}{l}49 \text { cases of poorly differentiated adenocarcinoma, } 23 \text { cases of mucinous } \\
\text { adenocarcinoma, } 72 \text { cases of moderately differentiated adenocarcinoma, and } \\
48 \text { cases of highly differentiated adenocarcinoma. And match the age. }\end{array}$ \\
\hline
\end{tabular}

\subsection{Data Analysis Methods}

Data collected were processed using SPSS19.0 and survival rates were analyzed using the kaplan-meier method and the chi-square test and kruskal-wallis rank sum test and the differences were significant when $\mathrm{P}<0.05$. 


\section{The Results}

\subsection{Data Analysis of Surgery, Complications and Local Recurrence in the Two Groups}

Through telephone inquiry, the return visit deadline is June 30,2019 . The return visit rate of group 1 and group 2 is $100 \%$, with no loss of visit. The pelvic local recurrence rates of group 1 and group 2 were 2.91\% (3/103) and 9.90\% (19/192), respectively, 3 years after surgery, with significant differences $(\mathrm{P}<0.05)$. Postoperative dysuria in group 1 and group 2 was $14.56 \%(15 / 103)$ and $5.21 \%(10 / 192)$, respectively, with significant difference $(\mathrm{P}<0.05)$. Postoperative sexual dysfunction in group 1 and group 2 was $12.50 \%(7 / 56)$ and $2.15 \%$ (2/93), respectively, with significant difference $(\mathrm{P}<0.05)$. The 5 -year survival rates of group 1 and group 2 were 54.37\% (56/103) and 51.56\% (99/192), respectively, with no significant difference $(\mathrm{P}>0.05)$. The liver metastasis rates of group 1 and group 2 were 26.21\% (27/103) and 29.17\% (56/192), respectively. Lung metastasis rates in group 1 and group 2 were $19.41 \% \quad(20 / 103)$ and $19.27 \% \quad(37 / 192)$, respectively. In group 1, 23 surgical specimens were pathologically found to have iliac perivascular lymph node metastasis, accounting for $22.33 \%$ of the cases undergoing iliac perivascular lymph node dissection. The perioperative status of group 1 and group 2 is shown in table 2 . It can be seen from table 2 that the operation time of the lateral cleaning group is longer than that of the TME group. The number of patients with dysuria after surgery was higher than that in the TME group, and the difference was significant, indicating that the rate of pelvic autonomic nerve injury in the lateral cleaning group was higher than that in the TME group. The amount of blood loss in the lateral cleaning group was higher than that in the TME group. Among the male patients in the two groups, there were 2 cases of erectile dysfunction in the TME group (2/93, 2.15\%), and 7 cases of erectile dysfunction in the lateral cleaning group $(7 / 56,12.50 \%)$, with significant difference. There was no significant difference in the number of postoperative rectal anastomotic fistula between group 1 and group 2 .

Table 2. Surgical conditions and complications of group 1 and group 2.

\begin{tabular}{lllll}
\hline Group & operation time (min) & dysuria (n) & erectile dysfunction (n) & Anastom-otic fistula (n) \\
\hline Group 2 & $162 \pm 33$ & 10 & $2.15 \%(2 / 93)$ & 6 \\
Group1 & $235 \pm 36$ & 15 & $12.50 \%(7 / 56)$ & 3 \\
P values & $\mathrm{P}<0.05$ & $\mathrm{P}<0.05$ & $\mathrm{P}<0.05$ & $\mathrm{P}>0.05$ \\
\hline
\end{tabular}

\subsection{5-year Survival Rate Analysis of the Two Groups}

In group 1, 56 patients $(56 / 103,54.37 \%)$ survived for 5 years, 20 patients died of lung metastasis and 27 patients died of liver metastasis. There were 99 patients $(99 / 192,51.56 \%)$ who survived for 5 years in the 2 groups, 37 patients died of lung metastasis and 56 patients died of liver metastasis, $\mathrm{P}<0.05$. There was no significant difference between the long-term survival rate of the 1 group and that of the 2 groups.

The survival functions of groups 1 and 2 are shown below

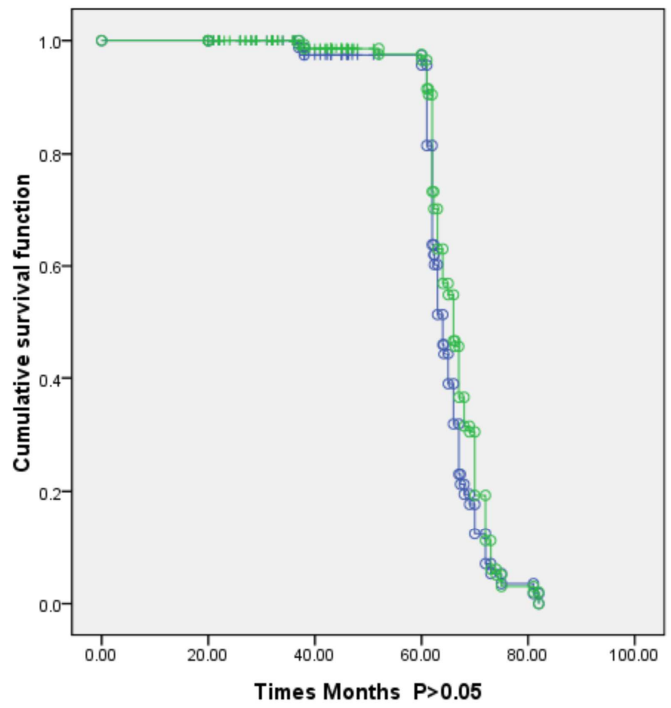

Figure 3. Table of survival functions of the lateral sweep group (group 1) and the TME group (group 2).

\section{Discuss}

\subsection{The Subperitoneal Reflux Route of Iliac Lymphatic Reflux for Rectal Cancer}

In recent years, Japanese scholars have summarized the study on the lymphatic reflux path of rectal cancer under peritoneal reflux. All rectal tumors are mainly reflux through the upper rectum vessels to the upper rectum lymph nodes, and then reflux through the lower mesenteric perivascular lymph nodes to the celiac trunk lymph nodes. There were bilateral periiliac lymphatic reflux routes in the rectal cancer under peritoneum reflux, and about $10 \%$ of the periiliac lymph nodes were positive under peritoneum reflux in the advanced stage. Anal tumors below the dentate line have a lower lymph node pathway that metastasized through the perineum, medial upper thigh segment or intermuscular lymphatic vessels along the iliac spine to the inguinal lymph node [14]. Now under peritoneal fold return mainstream colorectal surgery is before endoscopy assisted colorectal cancer resection and rectum holographic membrane excision (TME), but for advanced colorectal cancer under peritoneal fold return, especially with preoperative imaging tip iliac vessels weeks cases of lymph node enlargement, in addition to the long time history of radiation and chemotherapy after surgery, whether week of iliac blood vessels, lymphatic tissue expansion cleaning there is also debate [15]. At present, long-term preoperative chemoradiotherapy plays a significant role in the preoperative descending stage of peritonei in the advanced stage, but Akiyoshi et al found in a study that after neoadjuvant chemoradiotherapy, $66 \%$ of cases still showed positive lateral lymph node pathology, suggesting that neoadjuvant 
chemoradiotherapy cannot completely replace lateral lymph node dissection [16]. For the cases of periiliac lymph node enlargement indicated by MR after neoadjuvant chemoradiotherapy, the lymph node signals are uneven and the shape is irregular, so the lateral lymph and adipose tissue dissection should be accurately carried out. At the same time, the current research results also showed that the lateral lymph node metastasis of rectal tumor was related to the biological behavior of tumor cells, and the probability of lymph metastasis of low-differentiated adenocarcinoma and mucinous adenocarcinoma was relatively high. There was also a high probability of positive lymph nodes of tumor invading into the serous membrane of the rectum, and KRAS wild type, BRAF wild type and $\mathrm{P} 13 \mathrm{~K}$ wild type of rectal tumor were also prone to positive lymph nodes. For cases of perivascular iliac lymph node enlargement found by preoperative MR or intraoperative exploration, expanded lymph node dissection should be carried out accurately [17].

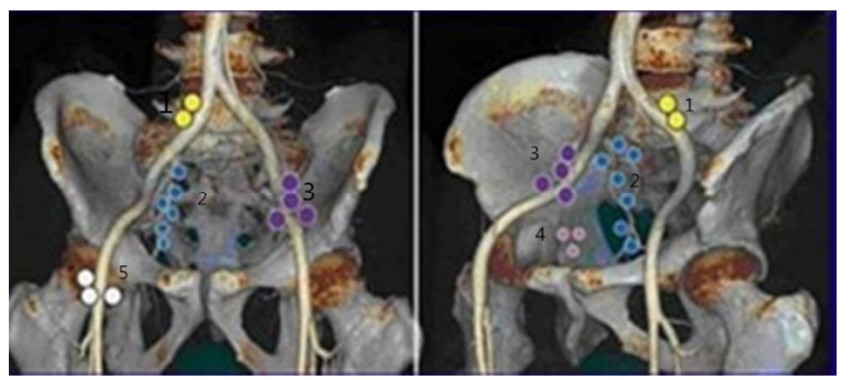

Figure 4. 1. Total iliac lymph nodes; 2. Internal iliac lymph nodes; 3. External iliac lymph nodes; 4. Obturator lymph nodes 5. Inguinal lymph nodes. [18]

\subsection{How to Accurately Evaluate Lymph Nodes by Rectal Magnetic Resonance}

There are no accurate and reliable method of rival preoperative lymph node metastasis in rectal tumor presence of iliac blood vessels weeks has confirmed that a study shows iliac blood vessels weeks lymph nodes is more than $10 \mathrm{~mm}$ in diameter may be there is a $10 \%$ chance to transfer, the existence of the lymph node is greater than $15 \mathrm{~mm}$ diameter has a $17 \%$ chance to appear tumor metastasis, lymph node more than $20 \mathrm{~mm}$ in diameter, will have a $35 \%$ chance the case of tumor metastasis [19]. But simply rely on the diameters of the lymph node to determine whether there is a tumor metastasis is not enough, in general the tumor involvement of lymph nodes is MR signal is not homogeneous, lymph node hilus structure shows vague, some scholars think should be symmetry with lymph node signal, can clearly show the possibility of a transfer for lymph node hilus structure [20-27]. Rectal MR can also be combined with ERUS(intra-rectal ultrasound). Ultrasound has its own unique feature in determining the nature of iliac perivascular lymph nodes. The metastatic lymph nodes showed uneven texture and invisible lymphatic hilum structure under ultrasound. PET - CT for rectal cancer currently also has reference value for the existence of lymph node metastasis, tumor metastasis of lymph nodes will appear in the accumulation of radioactive solid glucose, similar with the DWI (diffusion weighted imaging) can iliac blood vessels weeks about the existence of rectal cancer lymph node metastasis is helpful, but recently some scholars metastatic lymph nodes of PET judgment, sensitivity analysis, only $62 \%$ [28-35]. Recently, by combining artificial intelligence with tumor imaging and comparing big data of lymph node metastasis with tumor imaging of patients, the probability of judging lymph node metastasis is estimated to be $77.8 \%[31,13,36-43]$.

\subsection{Status of Neoadjuvant Comprehensive Therapy for Rectal Tumors with Subperitoneal Reflux}

Recently, Ishihara and Akiyoshi et al. respectively conducted follow-up studies on the incidence of pelvic local recurrence of patients who received adjuvant radiotherapy and chemotherapy, and found that 51.6-66\% of patients who received neoadjuvant radiotherapy and chemotherapy or TME alone had pelvic wall local recurrence within 5 years. This suggests that some cases still require periiliac lymphatic dissection after neoadjuvant therapy. After neoadjuvant comprehensive treatment, part of the mass of cases will shrink, and the radiation will cause pelvic regional tissue edema, the anatomical difficulty of the surgical field will increase after radiotherapy and chemotherapy, and the network like structure of pelvic autonomic nerve will be easily damaged during the operation, which will easily cause postoperative urination dysfunction in patients [13, 16, 41-47]. American scholars think that the internal iliac vessels distal segment lymph nodes are far beyond lymph nodes, distant lymph node as soon as possible after tumor metastasis, tiny transfer may be associated with the tumor cells in the circulatory system, with the passage of time, the tumor will colonize the viscera apart, far viscera tumor metastasis accounts for half of advanced colorectal cancer death. Therefore, American scholars do not recommend routine periiliac lymph node dissection, and advocate neoadjuvant comprehensive therapy [48]. In contrast, Sugihara's data analysis of multi-center rectal tumors in Japan showed that lateral lymph node dissection reduced the recurrence rate of pelvic cavity by $50 \%$ and the 5 -year survival rate of rectal cancer by $8 \%$ [49-51]. Meta-analysis of large samples by some Chinese scholars suggested that lateral lymphatic dissection did not increase the 5-year survival rate of rectal cancer patients, and increased the rate of pelvic autonomic nerve injury [52]. The reason for this is that although lateral lymphatic dissection removes local lymph nodes, it does not prevent distant metastasis of the tumor. Distant metastasis of tumor requires systemic chemotherapy to control. Some scholars believe that before neoadjuvant radiotherapy for advanced rectal tumor, several courses of systemic chemotherapy should be performed to solve the minor metastasis of tumor cell circulation system, and mFOLFOX6 and CAPOX are recommended [53].

\subsection{Whether Routine Lymph Node Dissection Should Be Performed Around the Iliac Vessels}

The European and American scholars and Japanese scholars 
for preventive lateral lymph node cleaning, there has been debate, a Japanese scholar of large colorectal cancer samples from 1991 to 1998 were retrospectively study, found that more than T2 tumor (invasion and muscle layer), and the nearer the anus, the greater the chance of a lateral lymph node metastasis, advanced low rectal cancer appear to $15.5 \%$ chance of lateral lymph node metastasis [16, 42, 54-59]. Therefore, most Japanese scholars performed preventive lateral lymph node dissection for advanced low rectal cancer. Different from Japan in the lymph node division of rectal Cancer, only the lymph nodes around the starting segment of internal iliac vessels are considered as regional lymph nodes, while the terminal internal iliac perivascular nodes, external iliac perivascular nodes and obturator lymph nodes are considered as distant lymph nodes. Most European and American scholars believe that distant lymph node metastasis is a systemic problem, and the removal of periiliac lymphatic adipose tissue cannot solve the problem of minimal metastasis in the circulatory system. Neoadjuvant chemoradiotherapy can replace lateral lymph node dissection [13, 41, 31, 60-64]. A study by heung-kwon et al. found that nearly $50 \%$ of rectal cancer patients were insensitive to neoadjuvant chemoradiotherapy, and $33 \%$ of rectal cancer cases after neoadjuvant chemoradiotherapy showed positive lateral lymph nodes [50]. Watanabe and Nagawa et al. conducted follow-up statistics on large samples after lateral lymph node dissection after neoadjuvant radiotherapy and chemotherapy respectively, and found no significant difference in 5-year overall survival rate of patients with lateral lymph node dissection compared with patients with only TME after neoadjuvant radiotherapy and chemotherapy. Lateral lymph node dissection did not improve the prognosis of patients with advanced rectal cancer. However, periiliac lymphatic dissection significantly increases the probability of urinary and reproductive system dysfunction in patients [65-66]. In summary, preoperative imaging of lateral lymph nodes is particularly important. Kusters et al. found that after neoadjuvant chemoradiotherapy, preoperative MR imaging of the rectum indicated that the incidence of local recurrence of the pelvic wall was much higher in patients with periiliac lymph nodes with a short diameter greater than $10 \mathrm{~mm}$ than that of patients with lymph nodes with a short diameter less than $5 \mathrm{~mm}[60,66,67]$. DWI (Diffusion weighted imaging) can describe the movement of water molecules in lymph nodes, and its sensitivity and specificity are both high for determining whether tumor metastasis exists in lateral lymph nodes. A study by Mizukami et al. showed that DWI can achieve sensitivity and specificity of $97 \%$ and $81 \%$ for metastatic lymph nodes. In our opinion, lateral lymph node dissection should be performed if the metastatic lateral lymph node is highly suspected by MR imaging after neoadjuvant chemoradiotherapy.

\subsection{Status of Neoadjuvant Comprehensive Therapy for Rectal Tumors with Subperitoneal Reflux}

Recently, Ishihara and Akiyoshi et al. respectively conducted follow-up studies on the incidence of pelvic local recurrence of patients who received adjuvant radiotherapy and chemotherapy, and found that 51.6-66\% of patients who received neoadjuvant radiotherapy and chemotherapy or TME alone had pelvic wall local recurrence within 5 years. This suggests that some cases still require periiliac lymphatic dissection after neoadjuvant therapy. After neoadjuvant comprehensive treatment, part of the mass of cases will shrink, and the radiation will cause pelvic regional tissue edema, the anatomical difficulty of the surgical field will increase after radiotherapy and chemotherapy, and the network like structure of pelvic autonomic nerve will be easily damaged during the operation, which will easily cause postoperative urination dysfunction in patients [13, 16, 41-47]. American scholars think that the internal iliac vessels distal segment lymph nodes are far beyond lymph nodes, distant lymph node as soon as possible after tumor metastasis, tiny transfer may be associated with the tumor cells in the circulatory system, with the passage of time, the tumor will colonize the viscera apart, far viscera tumor metastasis accounts for half of advanced colorectal cancer death. Therefore, American scholars do not recommend routine periiliac lymph node dissection, and advocate neoadjuvant comprehensive therapy [48]. In contrast, Sugihara's data analysis of multi-center rectal tumors in Japan showed that lateral lymph node dissection reduced the recurrence rate of pelvic cavity by $50 \%$ and the 5 -year survival rate of rectal cancer by $8 \%$ [49-51]. Meta-analysis of large samples by some Chinese scholars suggested that lateral lymphatic dissection did not increase the 5-year survival rate of rectal cancer patients, and increased the rate of pelvic autonomic nerve injury [52]. The reason for this is that although lateral lymphatic dissection removes local lymph nodes, it does not prevent distant metastasis of the tumor. Distant metastasis of tumor requires systemic chemotherapy to control. Some scholars believe that before neoadjuvant radiotherapy for advanced rectal tumor, several courses of systemic chemotherapy should be performed to solve the minor metastasis of tumor cell circulation system, and mFOLFOX6 and CAPOX are recommended [53].

\subsection{Lateral Lymph Node Dissection and Pelvic Autonomic Nerve Protection}

The biggest complication of lateral lymph node dissection is urinary and erectile dysfunction after pelvic autonomic nerve injury. The parasympathetic and sympathetic nerve fibers of the pelvic wall constitute the trunk branches and branches of the pelvic wall nerve, and the ends of the pelvic wall nerve are distributed in a network shape on both sides of the rectum. T10 each segment of the thoracic vertebra to L2 lumbar vertebra sends corresponding sympathetic and parasympathetic nerve fibers to form the upper inferior abdominal nerve network plexus, which is located in front of the abdominal segment of the aorta, and then runs down the double side of the submesenteric blood vessel, innervating the seminal vesicle and is related to ejaculation function $[42,50$, $56,58,68-70]$. The superior ventral inferior nerve network clusters on the promontory surface of the sacrum and sends out an inferior ventral nerve trunk to each side, which runs 
obliquely along the pelvic wall to both sides of the rectum. The most closely related to lateral lymph node dissection is the inferior ventral nerve network plexus. S2-4 sacral ganglion each sends out corresponding parasympathetic nerve fibers and nerve fibers from the inferior ventral nerve trunk on both sides of the rectum to form the inferior ventral nerve network plexus. The branches of the lower ventral inferior nerve network plexus the dorsal nerve of the penis passes through the lateral side of the rectum, the surface of the bladder, and the prostate gland to the cavernous body of the penis, responsible for erectile function of the penis. Lateral lymph node dissection along the far side of the iliac internal vessels of the skeletal vessels are prone to injury of the lower abdominal nerve network plexus, resulting in urinary and erectile dysfunction. If the vessels of the left colon are not retained when the submesenteric paravascularized lymphatic adipose tissue is swept, the severed submesenteric vessels should be slightly away from the roots, which is recommended to be more than $1.5 \mathrm{~cm}$ away from the roots to avoid the epigastric plexus. Endoscopy has the magnifying effect, and it should be marked by the presence of inferior abdominal nerve and inferior epigastric nerve network plexus under endoscopy when performing total mesorectal resection [43, 71-74]. In the bilateral rectal separation, the sacral rectal ligament should be separated from the rear first, and when extending to both sides, small steps should be taken to separate and search for the lower abdominal inferior neural network plexus, and free the medial rectal ligament in the lower abdominal inferior neural network plexus. In the ventral direction, the Denonvilliers membrane should be separated along the surface of the Denonvilliers membrane until the seminal vesicle level, and then the Denonvilliers membrane should be cut here to separate behind the lower abdominal nerve plexus, so as to avoid damaging the bladder branch, prostate branch and penis branch of the lower abdominal nerve plexus. If the tumor is located on one side and has invaded the lower abdominal nerve plexus on one side, it can be written to perform selective iliac vascular lymphatic dissection on one side, and attention must be paid to protect the contralateral nerve plexus, otherwise neurogenic bladder may occur, requiring lifelong fistula. Lateral lymph node dissection is a difficult operation, and general surgeons choose open surgery. However, in recent years, with the increasingly mature endoscopic surgery techniques, the number of lateral lymph node dissection under endoscopy gradually increased. After neoadjuvant chemoradiotherapy, radioactive inflammation of mesentery, blurred mesenteric space, increased blood loss, increased operation difficulty, and increased risk of pelvic autonomic nerve injury. The mechanical arm of the endoscopic instrument cannot be bent, and it is difficult to perform in the narrow space, which makes it difficult to carry out lymphatic dissection on the side of the endoscopic instrument. The robotic surgical system overcomes these shortcomings. The flexible mechanical arm and stable mechanical system provide guarantee for robotic lateral lymph node dissection [31, 38-42].

\subsection{Prognostic Factors Associated with Perivascular Lymph Node Metastasis in Iliac Rectal Cancer}

Japanese scholars believe that the external and perivascular iliac lymph nodes are distant lymph nodes, while European and American scholars believe that the distal perivascular lymph nodes of the internal iliac vessels are distant lymph nodes. Distant lymph node metastasis indicates that the tumor has progressed to systemic disease [43]. American scholars have reported in the literature that the incidence of distant organ metastases such as lung and liver in such cases of advanced rectal cancer reaches $50 \%$, and distant metastases are the biggest cause of death in advanced rectal cancer [43]. At this time, peri-iliac lymph node dissection could not improve the disease-free survival rate of the cases. In the cases with peri-iliac lymph node metastasis, half of the cases had metastases through the circulatory system within 5 years, and the most distant organs were the lungs, followed by the liver. In addition to the distant metastasis, local recurrence of advanced colorectal cancer basin wall important cause of death, basin once tumor local recurrence, the 5-year survival rate is only $20 \%$ [13], neoadjuvant chemoradiation greatly reduces the risk of recurrence of rectal cancer basin wall [44], before neoadjuvant chemoradiation promotion, with the literature, the basin of advanced colorectal cancer wall local recurrence rate can reach $25-50 \%$, neoadjuvant chemoradiation junior extension of rectal cancer wall local recurrence rate is still 5-10\% [50]. Part of peritoneal fold return under advanced cases of rectal cancer after neoadjuvant chemoradiation, week of iliac blood vessels, lymph node is intumescent, lymph nodes short diameter greater than $10 \mathrm{~mm}$, diffusion weighted imaging (DWI) can prompt the asymmetry signal in lymph nodes, this is the high risk factors, based on the magnetic resonance (NMR) locate precisely the cleared, but try not to be bilateral iliac blood vessels weeks lymph node cleaning at the same time, in order to reduce the rate of pelvic autonomic nerve damage [46].

\subsection{How to Reasonably Conduct Enlarged Lymph Node Dissection}

Rectum cancer above peritoneal retrograde is generally considered as colon cancer, and lymphatic dissection along iliac vessels is generally not possible. For rectum cancer with subperitoneal retroperitoneum, where MR does not suggest periiliac lymph node enlargement, total mesenteric resection (TME) is sufficient [45, 48, 49]. For anal cancer below the dentate line, neoadjuvant chemotherapy is usually followed by radical Miles surgery or local resection as appropriate. As the only means to completely remove positive lymph nodes and prevent recurrence, surgery should play an important role in the comprehensive treatment of advanced rectal cancer under peritoneum reflux and above the dentate line. Long time history of preoperative neoadjuvant chemoradiation can reach the effect of postoperative pathology negative, but new auxiliary synthesis of some cases the effect is not obvious, for this part of the case, we must repeatedly rectal mri assessment of tumor progression, and signal characteristics of week of 
iliac blood vessels and lymph nodes, its necessary weeks lymph node biopsy and cleaning the iliac blood vessels. Prophylactic lateral lymph node dissection, not supported by the author, has been promoted in Japan since the 1990s for advanced rectal cancer. But Kusters, etc, a study found that line neoadjuvant chemoradiation cases of pelvic local recurrence rate is only about $0.8 \%$ [67], lymphoid tissue removal of iliac blood vessels weeks process greatly increases the probability of the plexus injury basin wall neural network, the increasing cases of the probability of urogenital system dysfunction after surgery, and weeks of lymph node cleaning and iliac blood vessels failed to make the most of the cases of survival benefit. We believe that patients with highly suspected lateral lymph node metastasis should be selected for lateral lymph node dissection according to preoperative MR imaging. Diffusion weighted imaging (DWI) is recommended to determine the probability of lateral lymph node metastasis. During the operation, unilateral lateral lymph node dissection was performed according to the guidance of MRI images to reduce the incidence of neurogenic bladder. Preoperative MR imaging of the rectum should be combined with the short diameter of the iliac perivascular lymph node, which is less than $5 \mathrm{~mm}$, to consider the possibility of metastasis. Secondly, diffusion weighted imaging of lymph nodes should be combined with the lateral lymph node dissection if the possibility of lymph node metastasis is considered to be high, such as uneven signal and irregular shape [50]. This requires us to precisely clean the targeted lymph nodes during the operation. It is easy to damage the pelvic autonomic nerve and cause neurogenic bladder, erectile and ejaculation dysfunction. Therefore, caution should be taken in surgical indications. As for the scope of lateral lymph node dissection, NCCN suggests that the proximal lymph node of the internal iliac vessel should be dissected, and the internal iliac vessel should be skeletal. Under the existing endoscopic and robotic surgical system, the exposure and protection of the inferior ventral nerve trunk and the upper inferior ventral nerve network plexus can be easily achieved [51].

\subsection{Operative Points and Progress of Iliac Perivascular Lymph Node Dissection}

Due to the difficulty of surgery and the large amount of blood loss during the operation, open surgery is the most common method. In recent years, with the gradual maturity of endoscopic surgery, the number of cases of endoscopic iliac perivascular lymphatic dissection has gradually increased [52] Most surgical procedures of periiliac lymphatic dissection under endoscopy are TME, which is completed after rectal dissociation. After the distal rectum of the tumor is separated, the operation is carried out along the total iliac, internal iliac and external iliac vessels. Perivascular lymphatic dissection of the internal iliac vessels is the key point. Lymphatic vessels on both sides of the rectum and genitourinary organs converge to both sides of the pelvic cavity and are received by the lymph nodes around the internal iliac vessels $[16,42,56,75]$. The pelvic reticulum plexus on both sides of the ureter and rectum is located below the internal iliac blood vessel, with umbilical ligament in front, external iliac artery and vein on the outside, and iliopsoas muscle on the outside and rear. In the iliac arteriovenous bone mineralization, by using magnified view looking for lower abdomen cavity mirror and hypogastric nerve under power, under the show under the abdomen and abdominal nerve after power, then along the lower abdomen and hypogastric nerves under the power of the rear, along the iliac arteriovenous in separation of the iliac arteriovenous within each branch stripping lymphatic tissue, blood vessels, such as the bladder and blood vessels, pubic rectum bottom of blood vessels, such as cases for women with uterine artery. It is important to keep the bladder vessels on one side to prevent bladder dysfunction after surgery. The main focus of the separation of the common iliac artery and vein is to separate and retract the inferior abdominal nerve, and then to clear the surrounding adipose tissue. The genital femoral nerve should be avoided at the end of the dissection along the external iliac vessels. When separated along the lower part of the bladder, it enters the Retzius space of the pubic bladder. At this point, the obturator artery and vein and the obturator nerve can be seen. After a little dissociation, the obturator internus muscle can be seen. At present, due to the narrow space of periiliac lymphatic dissection under endoscope, the endoscope instrument is straight rod type, which cannot be bent and the operation is extremely difficult. Such as bleeding, can only be changed to open surgery. The emergence of the Da Vinci surgical robot operation method brings revolutionary change, surgical robot arm to rotate 360 degrees of the instrument, the operation stability of the mechanical arm, make it easier for the purpose of the operation, small operation space is no longer a week of iliac blood vessels lymph cleaning the obstacles, and intraoperative infrared detection system make the lymph node cleaning more accurate [60].

\section{Conclusion}

Advanced rectal cancer after neoadjuvant chemoradiation, on the basis of rectal MRI guidance, to highly suspected metastatic lymph node selective weeks of iliac blood vessels along the iliac blood vessels expand lymph cleaning, can reduce the patients of pelvic local recurrence 3 years, but increased the odds of a pelvic autonomic nerve damage, lateral lymph node cleaning the 5-year survival rate of the patients with no obvious benefits. Of course, there are still many shortcomings in this study, and the number of samples in this study is still insufficient, so the conclusions of this study can only be used for reference. Long-term follow-up and review are needed to provide more sufficient evidence based on the large sample size.

\section{References}

[1] Jayne DG, Thorpe HC, Copeland J, et al. Five-year follow-up of the Medical Research Council CLASICC trial of laparoscopically assisted versus open surgery for colorectal cancer [J]. Br J Surg, 2010, 97 (11): 1638-1645. 
[2] Hermanek $P$, Junginger $T$. The circumferential resection margin in rectal carcinoma surgery [J]. Tech Coloprotol, 2005, 26 (9): 193.

[3] Hohenberger W, Weber K, Matzel K, et al. Standardized surgery for colonic cancer: complete mesocolic excision and central ligation-technical notes and outcome [J]. Colorectal Dis, 2009, 11 (4): 354-364.

[4] Color II Study Group; Buunen M, Bonjer HJ, et al. COLOR II. A randomized clinical trial comparing laparoscopic and open surgery for rectal cancer [J]. Dan Med Bull 2009, 56 (2): 89-91.

[5] Haboubi N. Colonic surgery for cancer: a new paradigm [J]. Colorectal Dis, 2009, 11 (4): 333-334.

[6] Birgissona H, Talbackb M, Gunnarssona U, et al. Improved survival in cancer of the colon and rectum in Sweden [J]. Euro J Surg Oncol, 2005, 31 (8): 845-853.

[7] Quirke P, Steele R, Monson J, et al. Effect of the plane of surgery achieved on local recurrence in patients with operable rectal cancer: a prospective study using data from the MRC CR07 and NCIC-CTG CO16 randomised clinical trial [J]. Lancet, 009, 373: 821-828.

[8] Moriya Y. Treatment of lateral pelvic nodes metastases from rec-tal cancer: the future prospective [J]. G Chir, 2013, 34 (910): 245-248.

[9] Larson D, Marcello P, Larach S, et al. Surgeon Volume Does Not Predict Outcomes in the Setting of Technical Credentialing Results From a Randomized Trial in Colon Cancer [J]. Ann Surg, 2008, 248 (5): 746-750.

[10] Pramateftakis M. Optimizing colonic cancer surgery: high ligation and complete mesocolic excision during right hemicolectomy [J]. Tech Coloproctol, 2010, 14 (1 suppl): 49-51.

[11] Bertelsen C, Bols B, Ingeholm P, et al. Can the quality of colonic surgery be improved by standardisation of surgical technique with complete mesorectal excision? [J]. Colorectal Dis, 2011, 13 (10): 1123-1129.

[12] Chen SL, Bilchik AJ. More extensive nodal dissection improves survival for stages I to III of colon cancer: a population based study [J]. Ann Surg, 2006, 244 (4): 602-610.

[13] Nagawa H, Muto T, Sunouchi K, et al. Randomized, controlled trial of lateral node dissection vs. nerve- preserving resection in patients with rectal cancer after preoperative radiotherapy $[\mathrm{J}]$. Dis Colon Rectum, 2001, 44 (9): 1274-1280.

[14] den Dulk M, van de Velde C. Quality assurance in surgical oncology: the tale of the Dutch rectal cancer TEM trial [J]. J Surg Oncol, 2008, 97 (1): 5-7.

[15] Sammour T, Chang GJ. Lateral node dissection in low rectal cancer: Time for a global approach?[J]. Ann Surg, 2017, 266 (2): 208-209.

[16] Akiyoshi T, Ueno M, Matsueda K, et al. Selective lateral pelvic lymph node dissection in patients with advanced low rectal cancer treated with preoperative chemoradiotherapy based on pretreatment imaging [J]. Ann Surg Oncol, 2014, 21 (1): 189-196.

[17] Al-Sukhni E, Milot L, Fruitman M, et al. Diagnostic accuracy of MRI for assessment of T category, lymph node metastases, and circumferential resection margin involvement in patients with rectal cancer: A systematic review and meta-analysis [J].
Ann Surg Oncol, 2012, 19 (7): 2212-2223.

[18] Sun lingyu et al. Precise stratified treatment strategy for rectal cancer based on 2017ESMO guidelines, 27.08.2017

[19] Li XT, Sun YS, Tang L, et al. Evaluating local lymph node metastasis with magnetic resonance imaging, endoluminal ultrasound and computed tomography in rectal cancer: A meta-analysis [J]. Colorectal Dis, 2015, 17 (6): 129-135.

[20] Ishibe A, Ota M, Watanabe J, et al. Prediction of lateral pelvic lymph-node metastasis in low rectal cancer by magnetic resonance imaging [J]. World J Surg, 2016, 40 (4): 995-1001.

[21] Horne J, Bateman AC, Carr NJ, et al. Lymph node revealing solutions in colorectal cancer: should they be used routinely? [J]. J Clin Pathol, 2014, 67 (5): 383-388.

[22] Brown G, Richards CJ, Bourne MW, et al. Morphologic predictors of lymph node status in rectal cancer with use of high-spatial-resolution MR imaging with histopathologic comparison [J]. Radiology, 2003, 227 (2): 371-377.

[23] Ye Y, Liu T, Lu L, et al. Pre-operative TNM staging of primary colorectal cancer by (18) F-FDG PET-CT or PET: A meta-analysis including 2283 patients [J]. Int J Clin Exp Med, 2015, 8 (11): 21773-21785.

[24] Lee JY, Yoon SM, Kim JT, et al. Diagnostic and prognostic value of preoperative (18) F-fluorodeoxyglucose positron emission tomography/computed tomography for colorectal cancer: comparison with conventional computed tomography [J]. Intest Res, 2017, 15 (2): 208-214.

[25] Padhani AR, Liu GY, Mu- Koh D, et al. Diffusion- weighted magnetic resonance imaging as a cancer biomarker: consensus and recommendations [J]. Neoplasia, 2009, 11 (2): 102-125.

[26] Mizukami Y, Ueda S, Mizumoto A, et al. Diffusion- weighted magnetic resonance imaging for detecting lymph node metastasis of rectal cancer [J]. World J Surg, 2011, 35 (4): 895-899.

[27] Kinugasa $\mathrm{T}$, Akagi $\mathrm{Y}$, Ochi $\mathrm{T}$, et al. Lateral lymph-node dissection for rectal cancer: meta-analysis of all 944 cases undergoing surgery during 1975-2004 [J]. Anticancer Res, 2013, 33 (7): 2921-2927.

[28] Ueno M, Oya M, Azekura $K$, et al. Incidence and prognosticsignificance of lateral lymph node metastasis in patients with advanced low rectal cancer [J]. Br J Surg, 2005, 92 (6): 756-763.

[29] MERCURY Study Group, Shihab OC, Taylor F, et al. Relevance of magnetic resonance imaging-detected pelvic sidewall lymph node involvement in rectal cancer [J]. Br J Surg, 2 011, 98 (12): 1798-1804.

[30] Ogawa S, Hida JI, Ike H, et al. The important risk factor for lateral pelvic lymph node metastasis of lower rectal cancer is node-positive status on magnetic resonance imaging: study of the Lymph Node Committee of Japanese Society for Cancer of the Colon and Rectum [J]. Int J Colorectal Dis, 2016, 31 (10): 1719-1728.

[31] Ogawa S, Hida JI, Ike H, et al. Prediction of lateral pelvic lymph node metastasis from lower rectal cancer using magnetic resonance imaging and risk factors for metastasis: Multicenter study of the Lymph Node Committee of the Japanese Society for Cancer of the Colon and Rectum [J]. Int J Colorectal Dis, 2017, 32 (10): 1479-1487. 
[32] Gillies RJ, Kinahan PE, Hricak H. Radiomics: Images are more than pictures, they are data [J]. Radiology, 2016, 278 (2): 563-577.

[33] Parekh V, Jacobs MA. Radiomics: A new application from established techniques [J]. Expert Rev Precis Med Drug Dev, 2016, 1 (2): 207-226.

[34] Huang YQ, Liang CH, He L, et al. Development and validation of a radiomics nomogram for preoperative prediction of lymph node metastasis in colorectal cancer [J]. J Clin Oncol, 2016, 34 (4): $2157-2164$.

[35] Klang E. Deep learning and medical imaging [J]. J Thorac Dis, 2018, 10 (3): 1325-1328.

[36] Takahashi T, Ueno M, Azekura K, et al. Lateral node dissection and total mesorectal excision for rectal cancer [J]. Dis Colon Rectum, 2000, 43 (10): s59-s68.

[37] Yano H, Moran BJ. The incidence of lateral pelvic side all nodal involvement in low rectal cancer may be similar in Japan and the West [J]. Br J Surg, 2010, 95 (1): 33-49.

[38] Ryoma Y, Yoshifumi S, Hitoshi K, et al. Clinical significance of extramural tumor deposits in the lateral pelvic lymph node area in low rectal cancer: A retrospective study at two institutions [J]. Ann Surg Oncol, 2016, 23 (4): 552-558.

[39] Song JH, Jeong JU, Lee JH, et al. Preoperative chemoradiotherapy versus postoperative chemoradiotherapy for stage II-III resectable rectal cancer: A meta-analysis of randomized controlle trials [J]. Radiat Oncol J, 2017, 35 (3): 198-207.

[40] Ishibe A, Ota M, Watanabe J, et al. Prediction of lateral pelvic lymph-node metastasis in low rectal cancer by magnetic resonance imaging [J]. World J Surg, 2015, 40 (4): 1-7.

[41] Ishihara S, Kawai K, Tanaka T, et al. Oncological outcomes of lateral pelvic lymph node metastasis in rectal cancer treated with preoperative chemoradiotherapy [J]. Dis Colon Rectum, 2017, 60 (5): 469-476.

[42] Fujita S, Mizusawa J, Kanemitsu Y, et al. Mesorectal excision with or without lateral lymph node dissection for clinical stage II/III lower rectal cancer (JCOG0212) [J]. Ann Surg, 2017, 266 (2): 201-207.

[43] Sauer R, Becker H, Hohenberger W, et al. Preoperative versus postoperative chemoradiotherapy for rectal cancer. [J]. N Engl J Med, 2004, 351 (17): 1731-1740.

[44] Sauer R, Liersch T, Merkel S, et al. Preoperative versus postoperative chemoradiotherapy for locally advanced rectal cancer: results of the German CAO/ARO/AIO-94 randomized phase III trial after a median follow- up of 11 years [J]. J Clin Oncol, 2012, 30 (16): 1926-1933.

[45] Mallick S, Benson R, Haresh KP, et al. Neoadjuvant treatment intensification or adjuvant chemotherapy for locally advanced carcinoma rectum: The optimum treatment approach remains unresolved [J]. J Egypt Natl Canc Inst, 2015, 27 (4): 179-185.

[46] Stefano AD, Moretto R, Bucci L, et al. Adjuvant treatment for locally advanced rectal cancer patients after preoperative chemoradiotherapy: when, and for whom? [J]. Clin Colorectal Cancer, 2014, 13 (3): 185-191.

[47] Fujita S, Saito S, Moriya Y, et al. Postoperative morbidity andmortality after mesorectal excision with and without lateral lymph node dissection for clinical stage II or stage III lower rectal cancer (JCOG0212): results from a multicentre, randomised controlled, non-inferiority trial [J]. Lancet Oncol, 2012, 13 (6): 616-621.

[48] Akasu T, Iinuma G, Takawa M, et al. Accuracy of high resoluion magnetic resonance imaging in preoperative staging of rectal cancer [J]. Ann Surg Oncol, 2009, 16 (10): 2787-2794.2017, 25 (11): 801-806.

[49] Kim MJ, Kim TH, Kim DY, et al. Can chemoradiation allow for omission of lateral pelvic node dissection for locally advanced rectal cancer? [J]. J Surg Oncol, 2015, 111 (4): 459-464.

[50] Oh HK, Kang SB, Lee SM, et al. Neoadjuvantchemoradiotherapy affects the indications for lateral pelvic node dissection in mid/low rectal cancer with clinically suspected lateral node involvement: a multicenter retrospective cohort study [J]. AnnSurg Oncol, 2014, 21 (7): 2280-2287.

[51] Akiyoshi T, Matsueda K, sHiratsuka M, et al. Indications for lateral pelvic lymph node dissection based on magnetic resonan ceimaging before and after preoperative chemoradiotherapy in patients with advanced low-rectal cancer [J]. Ann Surg Oncol, 2015, 22 (suppl3): 614-620.

[52] Ogura A, Akiyoshi T, Nagasaki T, et al. Feasibility of laparoscopic total mesorectal excision with extended lateral pelvic lymph node dissectionfor advanced lower rectal cancer after preoperative chemoradiotherapy [J]. World J Surg, 2017, 41 (3): 868-875.

[53] Akasu T, Sugihara K, Moriya Y. Male urinary and sexual functions after mesorectal excision alone or in combination with extended lateral pelvic lymph node dissection for rectal cancer [J]. Ann Surg Oncol, 2009, 16 (10): 2779-2786.

[54] Col C, Hasdemir O, Yalcin E, et al. The assessment of urinary function following extended lymph node dissection for colorectal cancer [J]. Eur J Surg Oncol, 2005, 31 (3): 237-241.

[55] Fujita S, Yamamoto S, Akasu T, Moriya Y. Lateral pelvic lymph node dissection for advanced lower rectal cancer $[\mathrm{J}]$. Br J Surg, 2003, 90 (12): 1580-1585.

[56] Fujita S, Akasu T, Mizusawa J, et al. Postoperative morbidity andmortality after mesorectal excision with and without lateral lymph node dissection for clinical stage II or stage III lower rectal cancer (JCOG0212): results from a multicentre, randomised controlled, non-inferiority trial $[\mathrm{J}]$. Lancet Oncol, 2012, 13 (6): 616-621.

[57] Hasdemir O, Col C, Yalcin E, et al. Local recurrence and survival rates after extended systematic lymph-node dissection for surgical treatment of rectal cancer [J]. Hepatogastroenterology, 2005, 52 (62): 455-459.

[58] Kim JC, Takahashi K, Yu CS, et al. Comparative outcome between chemoradiotherapy and lateral pelvic lymph node dissection following total mesorectal excision in rectal cancer [J]. Ann Surg, 2007, 246 (5): 754-762.

[59] Kobayashi H, Mochizuki H, Kato T, et al. Outcomes of surgery alone for lower rectal cancer with and without pelvic sidewall dissection [J]. Dis Colon Rectum, 2009, 52 (4): 567-576.

[60] Kusters M, Beets GL, van de Velde CJ, et al. A comparison between the treatment of low rectal cancer in Japan and the Netherlands, focusing on the patterns of local recurrence [J]. Ann Surg, 2009, 249 (2): 229-235. 
[61] Kyo K, Sameshima S, Takahashi M, et al. Impact of autonomic nerve preservation and lateral node dissection on male urogenital function after total mesorectal excision for lower rectal cancer [J]. World J Surg, 2006, 30 (6): 1014-1019.

[62] Matsuoka H, Masaki T, Sugiyama M, et al. Impact of lateral pelvic lymph node dissection on evacuatory and urinary functions following low anterior resection for advanced rectal carcinoma [J]. Langenbecks Arch Surg, 2005, 390 (6): 517-522.

[63] Saito S, Fujita S, Mizusawa J, et al. Male sexual dysfunction after rectal cancer surgery: Results of a randomized trial comparing mesorectal excision with and without lateral lymph node dissection for patients with lower rectal cancer: Japan Clinical Oncology Group Study JCOG0212 [J]. Eur J Surg Oncol, 2016, 42 (12): 1851-1858.

[64] Watanabe T, Muro K, Ajioka Y, et al. Japanese Society for Cancer of the Colon and Rectum (JSCCR) guidelines 2016 for the treatment of colorectal cancer [J]. Int J Clin Oncol, 2018, 23 (1): 1-34.

[65] Watanabe T, Tsurita G, Muto T, et al. Extended lymphadenectomy and preoperative radiotherapy for lower rectal cancers [J]. Surgery, 2002, 132 (1): 27-33.

[66] Sugihara K, Kobayashi H, Kato T, et al. Indication and benefit of pelvic sidewall dissection for rectal cancer [J]. Dis Colon Rectum, 2006, 49 (11): 1663-1672.

[67] Akiyoshi T, Watanabe T, Miyata S, et al. Results of a Japanese Nationwide Multi-Institutional Study on Lateral Pelvic Lymph Node Metastasis in Low Rectal Cancer: Is It Regional or Distant Disease? [J]. Annals of Surgery, 2012, 255 (6): 1129-1134.

[68] Ogawa S, Itabashi M, Hirosawa T, et al. Lateral pelvic lymph node dissection can be omitted in lower rectal cancer in which the longest lateral pelvic and perirectal lymph node is less than $5 \mathrm{~mm}$ on MRI [J]. J Surg Oncol, 2014, 109 (3): 227-233.

[69] H Sato, K Maeda, M Maruta, et al. Who Can Get the Beneficial Effect from Lateral Lymph Node Dissection for Dukes C Rectal Carcinoma Below the Peritoneal Reflection? [J]. Dis Colon Rectum, 2006, 49 (10 Suppl): S3-S12.

[70] van der Pas MH, Haglind E, Cuesta MA, et al. Laparoscopic versus open surgery for rectal cancer (COLOR II): short-term outcomes of a randomised, phase 3 trial [J]. Lancet Oncol, 2013, 14 (3): 210-218.

[71] Bonjer HJ, Deijen CL, Abis GA, et al. A randomized trial of laparoscopic versus open surgery for rectal cancer [J]. N Engl J Med, 2015, 372 (14): 1324-1332.

[72] Liang JT. Technical feasibility of laparoscopic lateral pelvic lymph node dissection for patients with low rectal cancer after concurrent chemoradiation therapy [J]. Ann Surg Oncol, 2011, 18 (1): 153-159.

[73] Nagayoshi K, Ueki T, Manabe T, et al. Laparoscopic lateral pelvic lymph node dissection is achievable and offers advantages as a minimally invasive surgery over the open approach [J]. Surg Endosc, 2016, 30 (5): 1938-1947.

[74] Furuhata T, Okita K, Nishidate T, et al. Clinical feasibility of laparoscopic lateral pelvic lymph node dissection following total mesorectal excision for advanced rectal cancer [J]. Surg Today, 2015, 45 (3): 310-314.

[75] Hojo K, Vernava AM, Sugihara K, et al. Preservation of urine voiding and sexual function after rectal cancer surgery $[\mathrm{J}]$. Dis Colon Rectum, 1991, 34 (7): 532-539.

[76] Moriya Y, Sugihara K, Akasu T, et al. Nerve-sparing surgery with lateral node dissection for advanced lower rectal cancer [J]. Eur J Cancer, 1995, 31A (7-8): 1229-1232. 